Conclusions Here we used the $\mathrm{A} 2_{\mathrm{A}} / \mathrm{A} 2_{\mathrm{B}}$ dual antagonist AB928 to overcome adenosine-mediated suppression of CAR T cells. We found that AB928 enhanced important CAR T cell effector functions in the presence of the adenosine analogue, suggesting that combination therapy with $\mathrm{AB} 928$ may improve CAR T cell efficacy. This study was limited to in vitro experiments. To confirm the relevance of our findings, this combination therapy must be further investigated in an in vivo setting.

Disclosure Information M. Seifert: None. M. Benmebarek : None. B. Cadilha : None. J. Jobst: None. J. Dörr: None. T. Lorenzini: None. D. Dhoqina: None. J. Zhang: None. J. Zhang: None. U. Schindler: E. Ownership Interest (stock, stock options, patent or other intellectual property); Modest; Amgen Inc., Arcus Biosciences. Other; Significant; Arcus Biosciences. S. Endres: None. S. Kobold: B. Research Grant (principal investigator, collaborator or consultant and pending grants as well as grants already received); Significant; Arcus Biosciences.

\section{P08.06 COMBINING RIG-I-TARGETED IMMUNE ACTIVATION WITH CAR T CELL THERAPY INDUCES EFFICIENT TUMOR CONTROL IN MURINE PANCREATIC CANCER MODELS}

AM Senz*, SL Formisano, B Cadilha, T Lorenzini, S Endres, S Kobold, M Schnurr, LM König. Klinikum der universität münchen, München, Germany

\subsection{6/jitc-2021-ITOC 8.50}

Background The efficacy of chimeric antigen receptor (CAR) $\mathrm{T}$ cells against solid tumors remains unsatisfactory due to impaired trafficking of the CAR T cells into the tumor microenvironment (TME) and the presence of immunosuppressive factors and cells. 5'- triphosphate double-stranded RNA (3pRNA) is recognized by the intracellular pattern recognition receptor retinoic acid-induced gene I (RIG-I). RIG-I activates a downstream signaling cascade, triggering the expression of type I interferons (IFN), proinflammatory cytokines and chemokines enhancing immune surveillance in the TME. We hypothesized that priming the TME with RIG-I ligands increases the efficacy of CAR T cell therapy.

Materials and Methods T110299 pancreatic tumor cells (derived from a genetically-engineered Kras and p53 mutant murine PDAC model) were engineered to express murine epithelial cell adhesion molecule (EpCAM) and used to induce subcutaneous or orthotopic tumors in $\mathrm{C} 57 \mathrm{BL} / 6 \mathrm{~J}$ female mice. Mice bearing T110299 $\mathrm{EpCAM}^{+}$tumors were treated with intratumoral or i.v. injections of $3 p$-RNA followed by i.v. injection of syngeneic murine $\mathrm{T}$ cells that were retrovirally tranduced to express anti-EpCAM CARs. Three days after CAR $\mathrm{T}$ cell injection, immune cell composition and CAR $\mathrm{T}$ cell infiltration in the TME were assessed by flow cytometry. Additionally, tumor growth and survival were monitored.

Results Intratumoral injections of $3 p-R N A$ reshaped the myeloid immune compartment in the TME by significantly reducing suppressive polymorphonuclear-MDSC and macrophages while increasing Ly6Chigh inflammatory monocytes. Moreover, antigen-presenting cells, such as dendritic cells and macrophages, were activated as evidenced by increased MHC-I expression levels. This was paralleled by a significant increase in the infiltration of CAR T cells into the TME in the combination therapy group. Interestingly, anti-EpCAM CAR $\mathrm{T}$ cells alone failed to control the tumor growth of T110299
$\mathrm{EpCAM}^{+}$tumors, while monotherapy with 3p-RNA slightly delayed tumor growth in the subcutaneous model. Combination of 3p-RNA with anti-EpCAM CAR T cells induced a significant clinical benefit with tumor regression in $50 \%$ of the treated mice in the subcutaneous tumor model and prolonged survival in an orthotopic model.

Conclusions Remodeling the immunosuppressive TME using RIG-I ligands is a promising strategy for overcoming therapeutic resistance of CAR T cells in solid tumors, such as pancreatic cancer.

Funding The project was supported by the international doctoral program 'i-Target: Immunotargeting of cancer' funded by the Elite Network of Bavaria and the Stiftungen zu Gunsten der Medizinischen Fakultät.

Disclosure Information A.M. Senz: None. S.L. Formisano: None. B. Cadilha: None. T. Lorenzini: None. S. Endres: None. S. Kobold: None. M. Schnurr: None. L.M. König: None.

\section{P09 Young researcher session}

\section{P09.01 THE USE OF FDA APPROVED JAK, MTOR AND SRC INHIBITORS TO REGULATE T CELL-BISPECIFIC ANTIBODY-INDUCED CYTOKINE RELEASE WHILE NOT PREVENTING T CELL CYTOTOXICITY}

${ }^{1} \mathrm{G}$ Leclercq*, ${ }^{1} \mathrm{H}$ Haegel, ${ }^{1} \mathrm{~A}$ Schneider, ${ }^{1} \mathrm{~A}$ Giusti, ${ }^{1} \mathrm{~V}$ Pulko, ${ }^{2} \mathrm{~A}$ Toso, ${ }^{2} \mathrm{~T}$ Zimmermann, ${ }^{2} \mathrm{~L}$ Green, ${ }^{1} \mathrm{~N}$ Steinhoff, ${ }^{1} \mathrm{~J}$ Sam, ${ }^{1} \mathrm{M}$ Bacac, ${ }^{1} \mathrm{P}$ Umaña, ${ }^{1} \mathrm{C}$ Klein. ${ }^{1}$ Roche Innovation Center Zurich, Schlieren, Switzerland; ${ }^{2}$ Roche Innovation Center Basel, Basel, Switzerland

\subsection{6/jitc-2021-ITOC8.51}

Background $\mathrm{T}$ cell bispecific antibodies (TCBs) are potent $\mathrm{T}$ cell engagers, harboring a $2+1$ format with one binder to the $\mathrm{CD} 3 \epsilon$ chain and two binders to specific tumor antigens. Crosslinking of CD3 with tumor antigens triggers $\mathrm{T}$ cell activation and proliferation, cytokine release and tumor cell killing. TCB treatment is sometimes associated with safety liabilities due to on-target on-tumor or on-target off-tumor cytotoxicity and cytokine release. Off-tumor activity of the TCB may occur if the targeted tumor antigens are expressed on healthy cells, which may potentially result in tissue damages and compromise the patient's safety. Patients treated with TCBs may also experience a Cytokine Release Syndrome (CRS), characterized by fever, hypotension and respiratory deficiency and associated with the release of pro-inflammatory cytokines such as IL- 6 , TNF- $\alpha$, IFN- $\gamma$, and IL- $1 \beta$. Tyrosine kinases such as Src, mTOR and JAK1/2 are involved in downstream signaling pathways after engagement of the $T$ cell receptor.

Materials and Methods 52 FDA approved kinase inhibitors were screened in the presence of $\mathrm{T}$ cells activated on $\mathrm{CD} 3$ coated plates, mimicking TCB stimulation. Src, mTOR and JAK inhibitors were selected based on their capacity to prevent both, cytokine release and $\mathrm{T}$ cell proliferation. Using an in vitro model of target cell killing by human peripheral blood mononuclear cells stimulated with TCBs, we validated the effects of mTOR, JAK and Src kinase inhibitors on TCBinduced $\mathrm{T}$ cell activation, tumor cell killing and cytokine release. In vivo, the effect of mTOR, JAK and Src kinase inhibitors on TCB-induced cytokine release was confirmed in humanized NOD scid gamma (NSG) mice engrafted with human hematopoietic stem cells and treated with CD19-TCB. 
Results In line with previous reports for CAR-T cells, dasatinib (a src inhibitor) was found to fully switch off TCB-induced T cell functionality as well as the other src inhibitors bosutinib and ponatinib. In contrast, temsirolimus, sirolimus and everolimus (mTOR inhibitors) and ruxolitinib, baricitinib, tofacitinib, and fedratinib (JAK1/2 inhibitors) were found to more potently prevent TCB-induced cytokine release without blocking TCB-mediated target cell killing.

Conclusions These results provide evidence that the mechanisms of TCB-dependent cytokine release and tumor cell killing can be uncoupled. The FDA-approved mTOR and JAK1/2 inhibitors could potentially be used to mitigate CRS whereas the Src inhibitor dasatinib could rather stand as a potential antidote for on-target off-tumor activity or high-grade CRS.

Disclosure Information G. Leclercq: A. Employment (full or part-time); Modest; Roche. E. Ownership Interest (stock, stock options, patent or other intellectual property); Modest; Roche. H. Haegel: A. Employment (full or part-time); Modest; Roche. E. Ownership Interest (stock, stock options, patent or other intellectual property); Modest; Roche. A. Schneider: A. Employment (full or part-time); Modest; Roche. A. Giusti: A. Employment (full or part-time); Modest; Roche. V. Pulko: A. Employment (full or part-time); Modest; Roche. A. Toso: A. Employment (full or part-time); Modest; Roche. T. Zimmermann: A. Employment (full or part-time); Modest; Roche. L. Green: A. Employment (full or part-time); Modest; Roche. N. Steinhoff: A. Employment (full or part-time); Modest; Roche. J. Sam: A. Employment (full or part-time); Modest; Roche. M. Bacac: A. Employment (full or part-time); Modest; Roche. E. Ownership Interest (stock, stock options, patent or other intellectual property); Modest; Roche. P. Umaña: A. Employment (full or part-time); Modest; Roche. E. Ownership Interest (stock, stock options, patent or other intellectual property); Modest; Roche. C. Klein: A. Employment (full or part-time); Modest; Roche. E. Ownership Interest (stock, stock options, patent or other intellectual property); Modest; Roche.

\section{P09.02 EPIGENETIC MODULATION OF NEUROBLASTOMA ENHANCES T- AND NK CELL IMMUNOGENICITY VIA INDUCTION OF SURFACE EXPRESSION OF MHC CLASS I AND MICA/MICB}

\footnotetext{
${ }^{1,2} \mathrm{AM}$ Cornel ${ }^{*},{ }^{1,2} \mathrm{DAMH}$ van den Beemt, ${ }^{1,2} \mathrm{E}$ Dunnebach, ${ }^{1} \mathrm{MP}$ van Dierselhuis, 1,2S Nierkens. 'Princess Maxima Center, Utrecht, Netherlands; ${ }^{2}$ UMC Utrecht, Utrecht, Netherlands
}

10.1136/jitc-2021-ITOC8.52

Background Neuroblastoma (NBL) is the most common pediatric solid tumor and responsible for about $15 \%$ of all pediatric cancer deaths. The majority of high-risk (HR) patients suffers from relapse after intense therapy regimens, resulting in a 5 -year survival rate of only $40 \%$. Even though the potential of immune interference in HR-NBL is shown by the additive effect of anti-GD2 monoclonal antibody therapy to the treatment protocol, long-term follow-up studies reveal that the beneficial effect of immunotherapy diminishes over time. We hypothesize that this is a result of inadequate (adaptive) immune engagement caused by the extensive immunomodulatory capacity of HR-NBL and its microenvironment. One of the most remarkable immunomodulatory strategies of NBL tumors is the absence of MHC-I surface expression, thereby preventing cytotoxic $\mathrm{T}$ cell recognition and killing. MHC-I lacking cells are known to be subjected to NK cell mediated cytotoxicity, however, we have shown that NBL is able to evade this by temporary upregulating surface expression of MHC-I, thereby becoming temporarily more prone to $\mathrm{T}$ cell mediated cytotoxicity. The aim of this project is to identify pharmacological strategies to enhance adaptive immune activation and therewith immunogenicity of HR-NBL.

Materials and Methods FDA-approved drug libraries were screened to identify compounds enhancing MHC-I surface expression in NBL cell lines using high-throughput flow cytometry analyses optimized for adherent NBL cells. The effect of positive hits was subsequently confirmed in a panel of NBL patient-derived tumeroids. Alterations in the transcriptome and translatome upon incubation with compounds of interest were further studied to identify potential additional immunomodulatory effects in NBL. Ultimately, compound treated NBL cell lines and tumeroids were co-cultured with PRAME reactive tumor-specific $\mathrm{T}$ cells and healthy-donor NK cells to determine the in vitro effect on $\mathrm{T}$ - and $\mathrm{NK}$ cell cytotoxicity.

Results Drug library screening revealed MHC-I upregulation upon treatment of NBL cell lines and patient-derived tumeroids with multiple histon deacetylase inhibitors (HDACi). Further investigation of immunomodulatory effects of HDACi in NBL revealed enhanced expression of several additional players of the antigen presenting machinery, immunoproteasome expression, and MICA/MICB upregulation in NBL cells. We show that in untreated NBL cells, plasticity of MHC-I expression causes evasion of both NK- and T cell mediated cytotoxicity. Intriguingly, co-culture of NBL cells with tumor-specific $\mathrm{T}$ cells and healthy-donor NK cells upon treatment with the HDACi Entinostat resulted in enhanced in vitro T- and NK cell activation and cytotoxicity.

Conclusions We show pharmacological upregulation of MHCI, other antigen presenting machinery players, and the NKG2D ligands MICA/MICB upon HDACi in HR-NBL. Pretreatment of NBL with HDACi resulted in enhanced in vitro T- and NK cell mediated cytotoxicity, substantiating HDACi as a potential strategy to improve adaptive immune engagement and therewith immunogenicity to aid NBL treatment.

This work was supported by the Villa Joep Foundation [IWOV-Actief.51391.180034].

Disclosure Information A.M. Cornel: None. D.A.M.H. van den Beemt: None. E. Dunnebach: None. M.P. van Dierselhuis: None. S. Nierkens: None.

\section{P09.03 HYALURONIC ACID AS A NEW IMMUNOLOGIC ADJUVANT IN CANCER: DESIGN OF EFFECTIVE PREVENTIVE AND THERAPEUTIC VACCINATION STRATEGIES FOR HER2/NEU-POSITIVE BREAST TUMORS}

${ }^{1} \mathrm{D}$ Carpanese*, 'I Montagner, ${ }^{2} \mathrm{~A}$ Dalla Pietà, ${ }^{1} \mathrm{~V}$ Rossi, ${ }^{2} \mathrm{~A}$ Penna, ${ }^{2} \mathrm{G}$ Zuccolotto, ${ }^{2} \mathrm{G}$ Pasut, ${ }^{2} \mathrm{~A}$ Grigoletto, ${ }^{1} \mathrm{~A}$ Rosato. ${ }^{1}$ Veneto Institute of Oncology IOV-IRCCS, Padova, Italy; ${ }^{2}$ University of Padova, Padova, Italy

\subsection{6/jitc-2021-ITOC8.53}

Background The use of proteins as immunogens is attractive for the development of vaccines, but requires efficient adjuvants to overcome their weak immunogenicity. Recently, we investigated the potential of the TLR2/4 agonist hyaluronan (HA) as an immunological adjuvant for protein-based vaccines. ${ }^{1} 2$ Conjugation of $\mathrm{HA}$ to antigens strongly increased 